VOL. 56 (1997) [109-118]

\title{
PERIODIC-RECURRENT PROPERTY OF SOME CONTINUA
}

\author{
Janusz J. Charatonik and Wlodzimierz J. Charatonik
}

The equality between the closures of the sets of periodic and of recurrent points (called the periodic-recurrent property) is extended from mappings of a tree to mappings defined on a $\lambda$-dendroid obtained as a compactification of the complement of a finite subset of a tree provided that the components of the remainder have the same finite depth and each has the periodic-recurrent property.

\section{INTRODUCTION}

Let $X$ be a topological space. Throughout this paper $f: X \rightarrow X$ is assumed to be a continuous mapping of $X$ into itself. We denote by $\mathbb{N}$ the set of all positive integers, and by $\mathbb{R}$ the set of reals. For any $m \in \mathbb{N}$ let $f^{m}: X \rightarrow X$ denote the $m$-th iteration of $f$. A point $x$ of $X$ is said to be:

a periodic point of $f$ provided that there is $m \in \mathbb{N}$ such that $f^{m}(x)=x$;

a recurrent point of $f$ provided that for every neighbourhood $U$ of $x$ there is $m \in \mathbb{N}$ such that $f^{m}(x) \in U$.

The sets of periodic points, and of recurrent points of a mapping $f: X \rightarrow X$ will be denoted by $P(f)$ and $R(f)$ respectively. Thus we have

$$
P(f) \subset R(f) \subset X
$$

DEFINITION 1.2: A space $X$ is said to have the periodic-recurrent property (shortly $P R$-property) provided that for every mapping $f: X \rightarrow X$ the equality

$$
\operatorname{cl} P(f)=\operatorname{cl} R(f)
$$

holds.

Coven and Hedlund proved in [3] that the closed unit interval has the PR-property. The result has been extended to mappings on trees by Ye in [9].

Received 3rd September, 1996

Copyright Clearance Centre, Inc. Serial-fee code: 0004-9729/97 \$A2.00+0.00. 
THEOREM 1.4. (Ye) Every tree has the PR-property.

Recently Kato in [7] has shown that this theorem cannot be generalised to dendrites. Note that dendrites form a class of acyclic curves which are the nearest (in a sense) to trees because of arcwise connectedness. His method can also be applied to show that the Cantor fan, that is, the cone over the Cantor set, also does not have the PR-property, [2, Theorem 3.10]. These facts directed our study of the class of continua having the PR-property to acyclic but not arcwise connected curves. The simplest example of such continua is the $\sin (1 / x)$-curve

$$
S=\left\{(0, y) \in \mathbb{R}^{2}: y \in[-1,1]\right\} \cup\left\{(x, \sin (1 / x)) \in \mathbb{R}^{2}: x \in(0,1]\right\} .
$$

Thus, a question whether the $\sin (1 / x)$-curve $S$ has the PR-property was the starting point in our efforts to extend the result of Ye (Theorem 1.4) to more general classes of continua. Other results of Ye concerning dynamics of continuous functions on continua were also related to this particular curve. Let us recall that if $f$ is a homeomorphism of a hereditarily decomposable chainable continuum $X$ having finite depth (in the sense of Iliadis [6]), then equality (1.3) holds (even without taking closures, that is, $P(f)=R(f)$ ), see $[\mathbf{1 0}$, Corollary 3.5, p.92], where the term "Order" is used in the sense of depth. Our study goes further in this direction. Namely the PR-property is shown for each hereditarily decomposable and hereditarily unicoherent continuum $X$ having finite depth which is obtained from a tree $T$ by replacing a finite number of its points $q_{1}, \ldots, q_{n}$ of $T$ by some continua $Q_{1}, \ldots, Q_{n}$ of the same depth, each of which also has the PR-property. The replacment is made so that the union $\bigcup\left\{Q_{i}\right.$ : $i \in\{1, \ldots, n\}\}$ is the remainder in a compactification of $T \backslash\left\{q_{1}, \ldots, q_{n}\right\}$. As a very particular consequence of this result it follows that the $\sin (1 / x)$-curve has the PRproperty.

To present details of the construction of continua $X$ and of the proof of the main result, that is, of equality (1.3), we have to recall some preliminary concepts and assertions. They are contained in the next section.

\section{Preliminaries}

All spaces considered in this paper are assumed to be metric and separable. By a continuum we mean a compact connected space. For a continuum $X$ the symbol $C(X)$ stands for the family of all (nonempty) subcontinua of $X$, and $F_{1}(X)$ denotes the family of singletons of $X$. A locally connected continuum containing no simple closed curve is called a dendrite. A tree means a one-dimensional compact connected acyclic polyhedron. Thus every tree is a dendrite, but not conversely.

Recall that a continuum $X$ is said to be

hereditarily unicoherent provided that the intersection of any two subcontinua of $X$ is connected; 
hereditarily decomposable provided that every subcontinuum of $X$ is the union of two of its proper subcontinua;

a $\lambda$-dendroid if it is hereditarily unicoherent and hereditarily decomposable.

Given a $\lambda$-dendroid $X$ we denote by $\mathcal{P}(X)$ the family of all subcontinua $S$ of $X$ such that for each finite cover of $X$ the elements of which are subcontinua of $X$, the continuum $S$ is contained in a member of the cover. A (transfinite) well-ordered sequence (indexed with ordinals $\alpha$ ) of nondegenerate subcontinua $X_{\alpha}$ of a $\lambda$-dendroid $X$ is said to be normal provided that the following conditions are satisfied:

$$
\begin{aligned}
& X_{1}=X \\
& X_{\alpha+1} \in \mathcal{P}\left(X_{\alpha}\right) ; \\
& X_{\beta}=\bigcap\left\{X_{\alpha}: \alpha<\beta\right\} \quad \text { for each limit ordinal } \beta .
\end{aligned}
$$

The depth $k(X)$ of a $\lambda$-dendroid $X$ is defined as the minimum ordinal number $\eta$ such that the order type of each normal sequence of subcontinua of $X$ is not greater than $\eta$. The reader is referred to [6] and [8] for additional information related to this concept. The following three important statements concerning the depth will be needed in the present paper. For their proofs see [6, Theorems 1, 2 and 3, p.94-95].

Statement 2.4. For every two $\lambda$-dendroids $X$ and $Y$, if $Y \subset X$ then $k(Y) \leqslant k(X)$.

Statement 2.5. A $\lambda$-dendroid $X$ is locally connected (that is, it is a dendrite) if and only if $k(X)=1$.

Statement 2.6. If a $\lambda$-dendroid $Y$ is a continuous image of a $\lambda$-dendroid $X$, then $k(Y) \leqslant k(X)$.

\section{THE CONSTRUCTION}

A subcontinuum $Q$ of a continuum $X$ is said to be terminal provided that for every subcontinuum $K$ of $X$, if $K \cap Q \neq \emptyset$ then either $K \subset Q$ or $Q \subset K$. Aarts and van Emde Boas have proved [1, Theorem, p.35] that if $X$ is a locally compact, noncompact metric space, then each continuum is a remainder of $X$ in some compactification of $X$. The proof given in [1] let us formulate the result in an even stronger form, needed for our purposes.

THEOREM 3.1. (Aarts and van Emde Boas) If $X$ is a locally compact, noncompact metric space, then each continuum is a remainder of $X$ in some compactification of $X$ as a terminal subcontinuum of the compactification.

Let a tree $T$ and points $q_{1}, \ldots, q_{n}$ of $T$ be given for some positive integer $n$. Let $Q_{1}, \ldots, Q_{n}$ be continua. Choose in $T$ closed connected and mutually disjoint 
neighbourhoods $U_{1}, \ldots, U_{n}$ of points $q_{1}, \ldots, q_{n}$. Then for each $i \in\{1, \ldots, n\}$ the sets $U_{i} \backslash\left\{q_{i}\right\}$ are locally compact and noncompact. Thus applying Theorem 3.1 to each of them we construct in a standard way a compactification

$$
\gamma:\left(T \backslash\left\{q_{1}, \ldots, q_{n}\right\}\right) \rightarrow \gamma\left(T \backslash\left\{q_{1}, \ldots, q_{n}\right\}\right)
$$

such that:

(3.3) $X=\operatorname{cl} \gamma\left(T \backslash\left\{q_{1}, \ldots, q_{n}\right\}\right)$ is a continuum;

(3.4) the remainder $X \backslash \gamma\left(T \backslash\left\{q_{1}, \ldots, q_{n}\right\}\right)$ consists of $n$ components $Q_{1}, \ldots, Q_{n}$;

(3.5) for each index $i \in\{1, \ldots, n\}$ the continuum $Q_{i}$ is a terminal subcontinuum of $X$.

The following observation is a consequence of the definitions.

OBSERVATION 3.6. If the inserted continua $Q_{i}$ are $\lambda$-dendroids, then the resulting continuum $X$ defined by $(3.2)-(3.5)$ is a $\lambda$-dendroid, too.

Thus the concept of the depth $k(X)$ is well-defined for such $X$ (and for all subcontinua of $X$ ). The following proposition concerns the depth of some subcontinua of a continuum $X$ constructed in the above way.

THEOREM 3.7. Let a tree $T$ and points $q_{1}, \ldots, q_{n}$ of $T$ be given for some $n \in \mathbb{N}$. Take a compactification $\gamma$ as in (3.2) satisfying conditions (3.3)-(3.5) with some $\lambda$ dendroids $Q_{1}, \ldots, Q_{n}$ such that

(3.8) the depth $k\left(Q_{i}\right)$ is finite for each $i \in\{1, \ldots, n\}$.

Define the continuum $X$ as above by (3.2)-(3.5), and let $Y$ be a nondegenerate subcontinuum of $X$ such that, for some $i \in\{1, \ldots, n\}$

(3.9) $Y \cap Q_{i} \neq \emptyset \neq Y \backslash Q_{i}$.

Then

$$
k(Y) \geqslant 1+k\left(Q_{i}\right)
$$

ProOF: Terminality of the subcontinua $Q_{i}$ of $X$ (see condition (3.5)) implies by (3.9) that $Q_{i} \subset Y$. Therefore, for each finite cover of $Y$ whose elements are subcontinua of $Y$, the continuum $Q_{i}$ is contained in some element of the cover. Thus the family $\mathcal{P}(Y)$ (see tine definition of depth in the previous section) consists of the continua $Q_{j}$ intersecting $Y$ (thus contained in $Y$ by their terminality) and of their subcontinua; consequently, for each normal sequence in $Y$ we have $Y_{1}=Y$ and

$$
Y_{2} \in \bigcup\left\{C\left(Q_{j}\right) \backslash F_{1}\left(Q_{j}\right): j \in\{1, \ldots, n\} \text { with } Q_{j} \cap Y \neq \emptyset\right\},
$$

while each normal sequence in $Q_{i}$ starts with $Q_{i}$ and has its second term in $C\left(Q_{i}\right) \backslash$ $\left(F_{1}\left(Q_{i}\right) \cup\left\{Q_{i}\right\}\right)$, and thus the number of its terms is less by one than that of the corresponding normal sequence in $Y$. So (3.10) follows by (3.8) and by the definition of the depth. The proof is complete. 


\section{MAPPINGS}

This section plays an auxiliary role for proving main results contained in the next one. We start with the following proposition.

Proposition 4.1. Let $f: X \rightarrow X$ be a mapping of a continuum $X$ into itself. Then there exists exactly one subcontinuum $M(X, f)$ of $X$ such that

(4.2) $f \mid M(X, f): M(X, f) \rightarrow M(X, f) \quad$ is a surjection;

(4.3) $\quad P(f)=P(f \mid M(X, f)) \quad$ and $\quad R(f)=R(f \mid M(X, f))$;

(4.4) $M(X, f)$ is a maximal subcontinuum of $X$ satisfying (4.2).

Proof: Define $M(X, f)=\bigcap\left\{f^{m}(X): m \in \mathbb{N}\right\}$. Then $M(X, f)$ is a continuum as the intersection of an decreasing sequence of continua, and it satisfies conditions (4.2)-(4.4)

Therefore, when investigating mappings from a continuum $X$ into itself we see that the whole dynamics for $f$ is on $M(X, f)$, and thus we have the following corollary to Proposition 4.1.

COROLlary 4.5. When studying dynamics of a mapping $f: X \rightarrow X$ defined on a continuum $X$ we can assume without loss of generality that the mapping $f$ is a surjection.

Given a compact space $X$, we denote by $N(X)$ the set of points of $X$ at which $X$ is not locally connected. The following result is known (see [4, (3), p.28]).

THEOREM 4.6. (Engelking and Lelek) If $f$ is a mapping of a compact space $X$, then

$$
N(f(X)) \subset f(N(X)) \text {. }
$$

Let a tree $T$ and points $q_{1}, \ldots, q_{n}$ of $T$ be given for some $n \in \mathbb{N}$. Let $Q_{1}, \ldots, Q_{n}$ be continua. Take a compactification $\gamma$ as in (3.2) satisfying conditions (3.3)-(3.5) and define the continuum $X$ as previously by (3.2)-(3.5). Note that

$$
N(X)=\bigcup\left\{Q_{i}: i \in\{1, \ldots, n\}\right\} .
$$

Thus Theorem 4.6 implies the following assertion.

PROPOSITION 4.8. If a continuum $X$ is defined by conditions (3.2)-(3.5) and if a mapping $f: X \rightarrow X$ is a surjection, then

$$
\bigcup\left\{Q_{i}: i \in\{1, \ldots, n\}\right\} \subset f\left(\bigcup\left\{Q_{i}: i \in\{1, \ldots, n\}\right\}\right) .
$$

PROPOSITION 4.10. If the $\lambda$-dendroid $X$ is defined by conditions (3.2)-(3.5), if all continua $Q_{i}$ have the same finite depth, that is, if

$$
\text { there is } d \in \mathbb{N} \text { such that for each } i \in\{1, \ldots, n\} \text { we have } k\left(Q_{i}\right)=d \text {, }
$$


and if the mapping $f: X \rightarrow X$ is a surjection, then

(4.12) there are no indices $i, j \in\{1, \ldots, n\}$ such that $f\left(Q_{i}\right) \cap Q_{j} \neq \emptyset \neq f\left(Q_{i}\right) \cap$ $\left(X \backslash Q_{j}\right)$.

Further,

(4.13) there is no index $i \in\{1, \ldots, n\}$ such that $f\left(Q_{i}\right) \subset X \backslash \bigcup\left\{Q_{j}: j \in\right.$ $\{1, \ldots, n\}\}$.

PROOF: Suppose on the contrary that there are some indices $i$ and $j$ with (4.12). Then in applying Theorem 3.7 we can take $f\left(Q_{i}\right)$ for $Y$ in $(3.9)$, and we get $k\left(f\left(Q_{i}\right)\right) \geqslant$ $1+k\left(Q_{j}\right)$ by $(3.10)$. Since $k\left(Q_{i}\right) \geqslant k\left(f\left(Q_{i}\right)\right)$ by Statement 2.6 , the two inequalities lead by (4.11) to $d \geqslant 1+d$, a contradiction. To show the rest of the conclusion we suppose that there is an index $i$ satisfying (4.13), and without loss of generality we can take $i=1$. Then applying Proposition 4.8 we have by (4.9)

$$
\bigcup\left\{Q_{j}: j \in\{1, \ldots, n\}\right\} \subset \bigcup\left\{f\left(Q_{i}\right): i \in\{2, \ldots, n\}\right\} .
$$

So we see that $n-1$ continua $f\left(Q_{i}\right)$ have to cover $n$ continua $Q_{j}$, and thus one of the continua $f\left(Q_{i}\right)$ has to intersect at least two distinct continua $Q_{j}$, which is impossible by (4.12). The proof is complete.

Corollary 4.14. Let the $\lambda$-dendroid $X$ be defined by conditions (3.2)-(3.5) with (4.11). If the mapping $f: X \rightarrow X$ is a surjection, then

(4.15) for each $i \in\{1, \ldots, n\}$ there exists $j \in\{1, \ldots, n\}$ such that $f\left(Q_{i}\right)=Q_{j}$. Furthermore, the correspondence between indices $i$ and $j$ of (4.15) is one-to-one and it maps $\{1, \ldots, n\}$ onto itself.

ProOF: Take arbitrary $f\left(Q_{i}\right)$ and observe that it intersects some $Q_{j}$ by (4.13) of Proposition 4.10 , but it cannot intersect the complement of $f\left(Q_{j}\right)$ by (4.12). Thus

$$
f\left(Q_{i}\right) \subset Q_{j}
$$

We claim that

(4.17) there are no indices $i_{1}, i_{2} \in\{1, \ldots, n\}$ such that

$$
f\left(Q_{i_{1}}\right) \cap Q_{j} \neq \emptyset \neq f\left(Q_{i_{2}}\right) \cap Q_{j} \text { for some } j \in\{1, \ldots, n\}
$$

Indeed, if such indices $i_{1}, i_{2}$ and $j$ existed, then $f\left(Q_{i_{1}}\right) \cup f\left(Q_{i_{2}}\right) \subset Q_{j}$ by (4.16), and since all $Q_{i}$ 's have to be covered by their images according to inclusion (4.9) of Proposition 4.8, we would have $\bigcup\left\{Q_{i}: i \in\{1, \ldots, n\} \backslash\{j\}\right\} \subset \bigcup\left\{f\left(Q_{i}\right): i \in\right.$ $\left.\{1, \ldots, n\} \backslash\left\{i_{1}, i_{2}\right\}\right\}$, so $n-1$ continua $Q_{i}$ have to be covered by $n-2$ continua $f\left(Q_{i}\right)$, and consequently one of $f\left(Q_{i}\right)$ must intersect at least two distinct continua $Q_{i}$, which again is impossible by (4.12). Thus (4.17) is shown. Now (4.16) and (4.17) lead 
to $f\left(Q_{i}\right)=Q_{j}$ by (4.9). So (4.15) holds. Finally, (4.9) and (4.17) imply that the correspondence is both surjective and one-to one. The proof is complete.

Corollary 4.14 can be reformulated in the following form.

4.18. Corollary. Let the $\lambda$-dendroid $X$ be defined by conditions (3.2)(3.5) with (4.11). Then each surjective mapping $f: X \rightarrow X$ permutes the continua $Q_{i}$ for $i \in\{1, \ldots, n\}$.

Since $T \backslash\left\{q_{1}, \ldots, q_{n}\right\}$ has finitely many components that correspond in a one-to-one way to components of $X \backslash \bigcup\left\{Q_{i}: i \in\{1, \ldots, n\}\right\}$ (which coincide with arc components), Corollary 4.18 implies the next one.

COROLlaRY 4.19. Let the $\lambda$-dendroid $X$ be defined by conditions (3.2)-(3.5) with (4.11). Then each surjective mapping $f: X \rightarrow X$ permutes the components of the set $X \backslash \bigcup\left\{Q_{i}: i \in\{1, \ldots, n\}\right\}$, and consequently we have

$$
f\left(X \backslash \bigcup\left\{Q_{i}: i \in\{1, \ldots, n\}\right\}\right)=X \backslash \bigcup\left\{Q_{i}: i \in\{1, \ldots, n\}\right\} .
$$

\section{PERIODIC-RECURRENT PROPERTY}

As in the previous sections we consider a $\lambda$-dendroid $X$ obtained from a tree $T$ by replacing $n$ of its points $q_{1}, \ldots, q_{n}$ by $\lambda$-dendroids $Q_{1}, \ldots, Q_{n}$ of the same finite depth $d$ (see condition (4.11)) in such a way that they are components of the remainder under a compactification $\gamma$ (see (3.2)-(3.5)). For shortness, we put

$$
H=X \backslash \bigcup\left\{Q_{i}: i \in\{1, \ldots, n\}\right\} .
$$

Let $p: X \rightarrow T$ be the natural projection, that is, a mapping such that $p\left(Q_{i}\right)=\left\{q_{i}\right\}$ for each $i \in\{1, \ldots, n\}$ and $p \mid H=\gamma^{-1}: H \rightarrow\left(T \backslash\left\{q_{1}, \ldots, q_{n}\right\}\right)$ is a one-to-one mapping. Thus $p \mid H$ is a homeomorphism. Define a mapping $g: T \rightarrow T$ as follows. For each $i \in\{1, \ldots, n\}$ let $g\left(q_{i}\right)=p\left(f\left(p^{-1}\left(q_{i}\right)\right)\right)$, and $g(t)=p\left(f\left(p^{-1}(t)\right)\right)$ for each point $t \in T \backslash\left\{q_{1}, \ldots, q_{n}\right\}$. Since $f$ permutes the continua $Q_{i}$ according to Corollary 4.18, $g$ is well-defined, and its continuity is a consequence of the definition. Moreover, also by the definition of $g$, we have $p(f(x))=g(p(x))$ for each point $x \in X$, that is, the following diagram commutes.

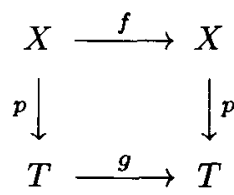

Commutativity of diagram (5.2) implies (using a simple induction argument) that, for each positive integer $m$, the diagram below commutes, too. 


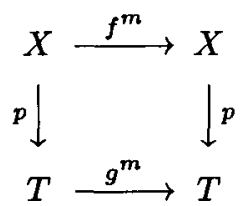

Moreover, by Corollaries 4.18 and 4.19 we have

$$
f^{m}\left(\bigcup\left\{Q_{i}: i \in\{1, \ldots, n\}\right\}\right)=\bigcup\left\{Q_{i}: i \in\{1, \ldots, n\}\right\} \text { and } f^{m}(H)=H .
$$

Proposition 5.4. For each point $x \in H$ we have the equivalences:

(5.5) $x \in P(f)$ is equivalent to $p(x) \in P(g)$;

(5.6) $x \in R(f)$ is equivalent to $p(x) \in R(g)$.

Proof: We start by showing (5.6). Let $x \in R(f)$, and let $U$ be an open neighbourhood of $p(x)$ in $T$. Thus there is $m \in \mathbb{N}$ such that $f^{m}(x) \in p^{-1}(U)$. So, $p\left(f^{m}(x)\right) \in p\left(p^{-1}(U)\right)=U$. Since by commutativity of diagram (5.3) we have $p \circ f^{m}=g^{m} \circ p$, it follows that $g^{m}(p(x)) \in U$, which shows one implication. Now let $p(x) \in R(g)$, and let $V$ be an open neighbourhood of $x$ in $X$. Since $H$ is open in $X$ by its definition, and since the partial mapping $p \mid H: H \rightarrow T \backslash\left\{q_{1}, \ldots, q_{n}\right\}$ is a homeomorphism, $p(V \cap H)$ is an open neighbourhood of $p(x)$. Thus by the assumption there is $m \in \mathbb{N}$ such that $g^{m}(p(x)) \in p(V \cap H)$. Since $g^{m} \circ p=p \circ f^{m}$ as previously, we have $p\left(f^{m}(x)\right) \in p(V \cap H)$, whence $p^{-1}\left(p\left(f^{m}(x)\right)\right) \in p^{-1}(p(V \cap H))=V \cap H \subset V$, and so $f^{m}(x) \in V$, that is, $x \in R(f)$. Equivalence (5.6) is shown.

To prove (5.5) we proceed analogously, omitting the consideration of neighbourhoods. Details are left to the reader. The proof is complete.

Now we are ready to show the main result of the paper.

5.7. TheOrEM. Let the $\lambda$-dendroid $X$ be obtained from a tree $T$ by replacing $n$ of its points $q_{1}, \ldots, q_{n}$ by $\lambda$-dendroids $Q_{1}, \ldots, Q_{n}$ of the same finite depth (4.11) using a compactification $\gamma$ with (3.2)-(3.5). If

(5.8) all continua $Q_{i}$ have the PR-property, then $X$ has the PR-property, too.

Proof: Let the mapping $f: X \rightarrow X$ be given. Consider the continuum $M(X, f)$ as in Proposition 4.1. If $M(X, f)$ is contained in some $Q_{i}$, then the conclusion follows from assumption (5.8). Otherwise the continuum $M(X, f)$ satisfies all the assumptions on $X$, so we can assume that $f$ is a surjection. According to Definition 1.2 and inclusion (1.1) we have to show that

$$
\operatorname{cl} R(f) \subset \operatorname{cl} P(f) .
$$

So, take $x \in \operatorname{cl} R(f)$ and a sequence of points $x_{k}$ of $R(f)$ tending to $x$, and consider three cases. 
CASE 1. $x \in H$. Since $H$ is an open subset of $X$, we may assume $x_{k} \in H$ for each $k \in \mathbb{N}$. By applying equivalence (5.6) of Proposition 5.4, we get $p\left(x_{k}\right) \in R(g)$. Hence $p(x) \in \operatorname{cl} R(g)$. Since the tree $T$ has the PR-property (Theorem 1.4) we have $p(x) \in \operatorname{cl} P(g)$. Since $x \in H$ implies $p(x) \in \gamma^{-1}(H)=T \backslash\left\{q_{1}, \ldots, q_{n}\right\}$ (which is an open subset of $T)$, there is a sequence of points $t_{k} \in\left(T \backslash\left\{q_{1}, \ldots, q_{n}\right\}\right) \cap P(g)$ tending to $p(x)$. By applying equivalence (5.5) of Proposition 5.4, we see that $p^{-1}\left(t_{k}\right) \in P(f)$. Since $p \mid H$ is a homeomorphism, the sequence $\left\{p^{-1}\left(t_{k}\right)\right\}$ tends to $p^{-1}(p(x))=x$, thereby $x \in \operatorname{cl} P(f)$.

CASE 2. $x \in Q_{i}$ and all $x_{k} \in Q_{i}$ for some $i \in\{1, \ldots, n\}$. By Corollary 4.18 there is $m \in \mathbb{N}$ such that $f^{m}\left(Q_{i}\right)=Q_{i}$. Since the condition $x_{k} \in R(f)$ is equivalent to the condition $x_{k} \in R\left(f^{m}\right)$ for each $m \in \mathbb{N}$ (see [5, Theorem I, p.126]), we get $x_{k} \in R\left(f^{m} \mid Q_{i}\right) \subset \operatorname{cl} R\left(f^{m} \mid Q_{i}\right)$, which implies that $x_{k} \in \operatorname{cl} P\left(f^{m} \mid Q_{i}\right)$, by assumption (5.8). Consequently, $x \in \operatorname{cl} P\left(f^{m} \mid Q_{i}\right) \subset \operatorname{cl} P(f)$.

CASE 3. $x \in Q_{i}$ for some $i \in\{1, \ldots, n\}$, and all $x_{k} \in H$. As previously we take $m \in \mathbb{N}$ such that $f^{m}\left(Q_{i}\right)=Q_{i}$, and again we have $x_{k} \in R\left(f^{m}\right)$ by Theorem I of [5, p.126], whence we infer by (5.6) of Proposition 5.4 that $p\left(x_{k}\right) \in R\left(g^{m}\right) \subset \operatorname{cl} R\left(g^{m}\right)$. By applying the PR-property for $T$ (Theorem 1.4) we see that $p\left(x_{k}\right) \in \operatorname{cl} P\left(g^{m}\right)$. Thus for each $k \in \mathbb{N}$ there is a sequence $\left\{x_{k}(r)\right\}$ of points of $H$ such that $x_{k}=\lim _{r \rightarrow \infty} x_{k}(r)$ and $p\left(x_{k}(r)\right) \in P\left(g^{m}\right)$. By equivalence (5.5) of Proposition 5.4, we get $x_{k}(r) \in P\left(f^{m}\right)$. Thus $x_{k} \in \operatorname{cl} P\left(f^{m}\right)$ for each $k \in \mathbb{N}$, and consequently $x \in \operatorname{cl} P\left(f^{m}\right) \subset \operatorname{cl} P(f)$.

Therefore inclusion (5.9) is shown, and thus $X$ has the PR-property. The proof is then complete.

CoRollary 5.10. The $\sin (1 / x)$-curve $S$ defined by (1.5) has the PR-property. QUESTION 5.11. Is the assumption (4.11) that all inserted continua $Q_{i}$ are of the same (finite) depth essential in Theorem 5.7?

Question 5.12. Can Theorem 5.7 be generalised to $\lambda$-dendroids $X$ in which the depth of some inserted continua $Q_{i}$ is infinite?

QUESTION 5.13. Is the condition that the number $n$ of the continua $Q_{i}$ is finite an essential assumption in Theorem 5.7? Under what conditions can the result be generalised to $\lambda$-dendroids $X$ in which the number of continua $Q_{i}$ is countable?

As a particular case of the above question we have the following.

QUESTION 5.14. Can Theorem 5.7 be extended to some continua $X$ obtained as compactifications of complements of closed countable subsets of trees?

All of the above questions are very particular cases of more general problems, which can be treated as a research program in the area, and which (at the present moment) seem to be rather far from any final solution. 
PROBLEM 5.15. What $\lambda$-dendroids have the PR-property?

Problem 5.16. Let an upper semicontinuous decomposition $\mathcal{D}$ of a continuum $X$ into continua (possibly degenerate) be given. Consider the following three conditions:

(5.17) the continuum $X$ has the PR-property;

(5.18) all (or some) members of the decomposition $\mathcal{D}$ have the PR-property;

(5.19) the decomposition space $X / \mathcal{D}$ has the PR-property.

What are interrelations between these conditions?

In particular, the following question is related to Theorem 5.7 and is especially interesting.

QUESTION 5.20. Under what assumptions do conditions (5.18) and (5.19) imply (5.17)?

\section{REFERENCES}

[1] J.M. Aarts and P. van Emde Boas, 'Continua as remainders in compact extensions', Nieuw Arch. Wisk. 15 (1967), 34-37.

[2] J.J. Charatonik, 'On sets of periodic and of recurrent points', (preprint).

[3] E.M. Coven and G.A. Hedlund, ' $\bar{P}=\bar{R}$ for maps of the interval', Proc. Amer. Math. Soc. 79 (1980), 316-318.

[4] R. Engelking and A. Lelek, 'Cartesian products and continuous images', Colloq. Math. 8 (1961), 27-29.

[5] P. Erdös and A. H. Stone, 'Some remarks on almost periodic transformations', Bull. Amer. Math. Soc. 51 (1945), 126-130.'

[6] S. Iliadis, 'On classification of hereditarily decomposable continua', Moscow Univ. Math. Bull. 29 (1974), 94-99.

[7] H. Kato, 'A note on periodic points and recurrent points of maps of dendrites', Bull. A ustral. Math. Soc. 51 (1995), 459-461.

[8] L. Mohler, 'The depth in tranches in $\lambda$-dendroids', Proc. Amer. Math. Soc. 96 (1986), 715-720.

[9] X.D. Ye, 'The centre and the depth of the centre of a tree map', Bull. Austral. Math. Soc. 48 (1993), 347-350.

[10] X.D. Ye, 'The dynamics of homeomorphisms of hereditarily decomposable chainable continua', Topology Appl. 64 (1995), 85-93.

\author{
Mathematical Institute \\ University of Wrocław \\ Pl. Grunwaldzki 2/4 \\ 50-384 Wrocław \\ Poland \\ e-mail: jjc@hera.math.uni.wroc.pl \\ jjc@gauss.matem.unam.mx
}

\author{
Departamento de Matemáticas \\ Facultad de Ciencias \\ Ciudad Universitaria \\ 04510 Mexico, D.F. \\ México \\ e-mail: wjcharat@hera.math.uni.wroc.pl \\ wjcharat@lya.fciencias.unam.mx
}

